Pacific Journal of Mathematic 


\section{COMPARISON THEOREMS FOR PARABOLIC FUNCTIONAL INEQUALITIES}

\section{RAYMOND REDHEFFER AND WOLFGANG WALTER}

Differential inequalities containing functionals are assuming an increasing importance in problems of biomathematics, mathematical medicine, chemistry, heat flow and population growth. Many of these applications lead to an equation which is of parabolic structure, in the sense that the equation would be parabolic if the functional in it were replaced by a known function. One way in which a functional arises in such equations is through a Volterra type memory term, which takes account of the past history of the process.

We shall present a number of comparison inequalities for parabolic functional operators. These can be used to answer questions pertaining to uniqueness, monotonicity, stability and qualitative behavior with the same simplicity and directness as has long been available in the purely parabolic case. As an application, we obtain new results on the behavior of strongly coupled systems.

The particular formulation adopted here has its historical origin in the concept of monotone operator, as introduced by Collatz [1]. Without getting involved in technical details, let us suppose that we have an "interior operator" $P$ and a "boundary operator" $R$ defined for functions in some suitable class $Z$. The pair $(P, R)$ is said to be monotone in the sense of Collatz if the implication

$$
P u \leqq P v, R u \leqq R v \Longrightarrow u \leqq v
$$

holds for $u, v \in Z$. In the case of an unbounded region one may need a growth condition such as $\sup (u-v)<\infty$. Further generalization can be achieved by allowing an interior error $\delta$ and a boundary error $\varepsilon$. The implication is now

$$
P u-P v \leqq \delta, R u-R v \leqq \varepsilon, \sup (u-v)<\infty \Longrightarrow u-v \leqq \rho,
$$

where the bound $\rho$ is supposed to be effectively computable in terms of $\delta$ and $\varepsilon$. The usefulness of such results in the study of uniqueness, stability, numerical estimation and existence is well known $[2,12,13]$.

The above formulation (1) is one-sided, in the sense that there is a one-sided bound in both hypothesis and conclusion. Many important results (such as Harnack inequalities, for example) do not hold in the one-sided case, but do hold when the estimates are two- 
sided. The two-sided version of (1) is

$$
\begin{aligned}
|P u-P v| & \leqq \delta,|R u-R v| \leqq \varepsilon, \sup |u-v|<\infty \\
& =|u-v| \leqq \rho .
\end{aligned}
$$

Even in the case of purely parabolic operators, one-sided estimates hold only for systems satisfying certain monotonicity conditions, while two-sided bounds can be obtained for a much larger class of equations.

The following results are formulated in the style (2). The necessary additional assumptions needed for corresponding results (1) are, roughly speaking, a quasimonotonicity condition with respect to $u$ and a monotonicity condition with respect to $u(\cdot)$. The modifications in the proofs are easy and are left to the reader.

We point out that existence theorems for the type of problems discussed here can be proved, using essentially the same methods as in the purely parabolic case (a priori estimates combined with fixedpoint theorems or Leray-Schauder degree theory). We shall come back to this matter in another paper.

2. Notation. We use $|\cdot|$ for the Euclidean norm; the argument can be any finite-dimensional vector or matrix. Points of $\boldsymbol{R}^{n+1}$ are written in the form $(x, t)$ with $x \in \boldsymbol{R}^{n}$ and $t \in \boldsymbol{R}$. The letter $G$ denotes a nonempty subset of $\boldsymbol{R}^{n+1}$ which has the following two properties:

(i) $\inf t=0, \sup t=T>0$ for $(x, t) \in G$.

(ii) If $(x, t) \in G$ then a half-neighborhood of form

$$
\{(\xi, \tau): t-\alpha<\tau \leqq t,|x-\xi|<\alpha\}
$$

with $\alpha=\alpha(x, t)>0$ is also in $G$. We define $\Gamma=G_{0}-G$ where $G_{0}$ is a given closed set containing $\bar{G}$ and we use the terminology

$$
G=\text { parabolic interior, } \Gamma=\text { parabolic boundary. }
$$

As explained in [9], introduction of $G_{0}$ is needed to ensure that expressions like $u(2 x, t-1)$ are defined in $G$. In purely parabolic problems $G_{0}=\bar{G}$ and $\Gamma$ is the parabolic boundary in the ordinary sense.

At certain points $(x, t) \in \Gamma$ one can define an inner normal $\nu(x, t)$ by use of a sequence of points $\left(x_{j}, t\right) \in G$; see [13; $\left.\S 31\right]$. A point $(x, t) \in \Gamma$ is called a boundary point of Type $\mathrm{I}$ if the normal derivative does not occur in the boundary condition at $(x, t)$. All points in $\Gamma-\bar{G}$ are of Type $I$, as are all points $(x, 0) \in \Gamma$. A point $(x, t) \in$ $\Gamma$ is of Type II if the boundary condition involves the normal derivative at $(x, t)$; this implies of course that $\nu(x, t)$ exists. 
For functions $w: G_{0} \rightarrow \boldsymbol{R}^{m}$ we assign to $w_{t}, w_{\nu}, w_{x}, w_{x x}$ the meanings that have long been traditional in parabolic problems. Thus, $w_{t}$ is a one-sided derivative from below with respect to $t, w_{\nu}$ is the inner normal derivative, $w_{x}$ is the gradient, and $w_{x x}$ the Hessian. At a given point $(x, t)$ where these expressions exist we have

$$
w_{t} \in \boldsymbol{R}^{m}, w_{\nu} \in \boldsymbol{R}^{m}, w_{x} \in\left(\boldsymbol{R}^{n}\right)^{m}, w_{x x} \in\left(S^{n}\right)^{m}
$$

where $S^{n}$ denotes the set of real symmetric $n$ by $n$ matrices. Existence of $w_{x x}$ means that each coordinate of $w_{x}$ is differentiable in the $n$-variable sense; mere existence of the second partial derivatives is not enough.

We conclude this summary of notation by giving two definitions and a notational convention.

Definition 1. The class $Z^{m}$ of admissible functions is the class of functions $w: G_{0} \rightarrow \boldsymbol{R}^{m}$ such that

(i) $w$ is continuous in $G_{0}$,

(ii) $w_{t}, w_{x}$ and $w_{x x}$ exist in $G$.

Definition 2. For $z \in \boldsymbol{R}^{m}, \phi \in Z^{1}$ and $w \in Z^{m}$, respectively,

$$
\begin{aligned}
& \|\boldsymbol{z}\|=\left(\left|z^{1}\right|,\left|z^{2}\right|, \cdots,\left|z^{m}\right|\right), \\
& |\phi|_{t}=\sup \left\{|\boldsymbol{\phi}(\xi, \tau)|:(\xi, \tau) \in G_{0}, \tau \leqq t\right\}, \\
& \|w\|_{t}=\left(\left|w^{1}\right|_{t},\left|w^{2}\right|_{t}, \cdots,\left|w^{m}\right|_{t}\right) .
\end{aligned}
$$

This use of the subscript $t$ should be distinguished from its use to denote partial differentiation, as in $u_{t}$.

Notational convention. The letters $u$ and $v$ denote functions of class $Z^{m}$. The letters $\delta, \varepsilon, \rho$ denote continuous functions $(-\infty, T] \rightarrow \boldsymbol{R}^{m}$ which are constant for $t \leqq 0$.

This convention is introduced to eliminate unnecessary clutter in the statements of our theorems. The functions $\delta, \varepsilon, \rho$ are given in the first instance as functions $[0, T] \rightarrow \boldsymbol{R}^{m}$, but are extended to $(-\infty, 0)$ so they will be defined on $G_{0}$.

3. Inequalities and monotonicity. Inequalities between vectors of $\boldsymbol{R}^{m}$ are interpreted componentwise, but inequalities between symmetric $n$ by $n$ matrices are interpreted by means of quadratic forms. Thus, if $z \in \boldsymbol{R}^{m}$ and $\bar{z} \in \boldsymbol{R}^{m}$, then

$$
z \leqq \bar{z} \Longleftrightarrow z^{i} \leqq \bar{z}^{i}, z<\bar{z} \Longleftrightarrow z^{i}<\bar{z}^{i}(i=1,2, \cdots, m) \text {. }
$$

But if $s \in S^{n}$ and $\bar{s} \in S^{n}$, then 


$$
s \leqq \bar{s} \Longleftrightarrow \xi(s-\bar{s}) \xi \leqq 0 \text { for } \xi \in \boldsymbol{R}^{n},
$$

where $\xi(s-\bar{s}) \xi$ denotes the quadratic form $\sum_{i, j=1}^{n}\left(s_{i j}-\bar{s}_{i j}\right) \xi_{i} \xi_{j}$. Inequalities are extended to functions by the usual pointwise definitions, and we set $\boldsymbol{R}_{+}^{m}=\left\{z: z \in \boldsymbol{R}^{m}, z \geqq 0\right\}, R_{+}=R_{+}^{1}$.

In general a function $\psi$ is increasing if

$$
s \leqq \bar{s} \Longrightarrow \psi(s) \leqq \psi(\bar{s}),
$$

where the ordering is that which is appropriate to the category of the variable. For example, if $\psi$ is a function $S^{n} \rightarrow \boldsymbol{R}$, then the inequality $s \leqq \bar{s}$ is a matrix inequality as introduced above, while if $\psi$ is a function $\boldsymbol{R}^{m} \rightarrow \boldsymbol{R}^{m}$ both inequalities are vector inequalities in $\boldsymbol{R}^{m}$. A function $\rho:[0, T] \rightarrow \boldsymbol{R}^{m}$ is increasing if each coordinate $\rho^{k}$ is; this is consistent with the above conventions.

In nonlinear problems it is essential to specify the "base value" at which the monotony inequality is required. This is accomplished here by use of an arrow which also specifies the relevant variable. For example, if $\phi$ is a function $G \times \boldsymbol{R}^{m} \times \boldsymbol{R}^{n} \times S^{n} \rightarrow \boldsymbol{R}$ the statement

$$
\phi\left(x, t, u, u_{x}^{k}, u_{x x}^{k} \uparrow\right) \text { is monotone }
$$

means that

$$
\phi\left(x, t, u, u_{x}^{k}, u_{x x}^{k}+s\right) \geqq \phi\left(x, t, u, u_{x}^{k}, u_{x x}^{k}\right) \geqq \phi\left(x, t, u, u_{x}^{k}, u_{x x}^{k}-s\right)
$$

for $s \in S^{n}, s \geqq 0$. Here $u$ is a specific function $u \in Z^{m}$ and $u, u_{x}^{k}, u_{x x}^{k}$ are abbreviations for

$$
u(x, t), \quad u_{x}^{k}(x, t), \quad u_{x x}^{k}(x, t),
$$

respectively. The inequality is required for $(x, t) \in G$.

In a like fashion, if $\phi$ is a function $\Gamma \times \boldsymbol{R}^{m} \times \boldsymbol{R} \rightarrow \boldsymbol{R}$, the statement

$$
\dot{\phi}\left(x, t, u, u_{*}^{k} \uparrow\right) \text { is monotone }
$$

means that

$$
\dot{\phi}\left(x, t, u, u_{\nu}^{k}+s\right) \geqq \phi\left(x, t, u, u_{\nu}^{k}\right) \geqq \phi\left(x, t, u, u_{\nu}^{k}-s\right)
$$

for $s \in R, s \geqq 0$. Here $u$ is a specific function $u \in Z^{m}$, and $u$ and $u_{\nu}^{k}$ are abbreviations for $u(x, t)$ and $u_{\nu}^{k}(x, t)$, respectively. The inequality is required for $(x, t) \in \Gamma$. Naturally, similar conventions apply if $\phi$ has an extra argument $u(\cdot)$, as in the sequel.

Throughout this paper, monotonicity conditions are prescribed at the argument $u$ and continuity conditions at the argument $v$, where $u$ and $v$ are specific functions entering into the statements of 
our theorems.

We conclude by introducing the following definition:

Definition 3. The function $\omega: \boldsymbol{R} \times \boldsymbol{R}^{m} \times \boldsymbol{R}^{m} \rightarrow \boldsymbol{R}^{m}$ is quasimonotone increasing if the conditions

$$
\bar{y} \leqq y, \quad \bar{y}^{k}=y^{k}, \quad \bar{z} \leqq z, \quad \bar{z}^{k}=z^{k}
$$

together imply $\omega_{k}(t, \bar{y}, \bar{z}) \leqq \omega_{k}(t, y, z)$ for $t \in R$ and for $k=1,2, \cdots, m$.

A similar definition applies to the function $\gamma(t, y)$, which does not depend on $z$.

4. A preliminary result. Throughout the sequel we shall be concerned with operators of the form

$$
P=\left(P_{1}, P_{2}, \cdots, P_{m}\right), \quad R=\left(R_{1}, R_{2}, \cdots, R_{m}\right),
$$

where

$$
\left\{\begin{array}{l}
P_{k} u=u_{t}^{k}-f_{k}\left(x, t, u, u_{x}^{k}, u_{x: k}^{k} \uparrow, u(\cdot)\right) \\
R_{k} u=u^{k}-g_{k}\left(x, t, u, u_{\nu}^{k} \uparrow, u(\cdot)\right) .
\end{array}\right.
$$

Here $u(\cdot)$ denotes the function itself, as a member of $Z^{m}$, while the other arguments of $f_{k}$ and $g_{k}$ stand for the values at $(x, t)$. Thus, $f_{k}$ and $g_{k}$ are respectively of forms

$$
G \times \boldsymbol{R}^{m} \times \boldsymbol{R}^{n} \times S^{n} \times Z^{m} \longrightarrow \boldsymbol{R}, \quad \Gamma \times \boldsymbol{R}^{m} \times \boldsymbol{R} \times \boldsymbol{Z}^{m} \longrightarrow \boldsymbol{R} .
$$

It is understood that the argument $u_{\nu}^{k}$ is absent from $g_{k}$ at points of Type I. At these points, no monotonicity condition for $g_{k}$ is required.

As continuity conditions we assume

$$
\left\{\begin{array}{c}
\left(\operatorname{sgn} z^{k}\right)\left[f_{k}\left(x, t, v+z, v_{x}^{k}, v_{x x}^{k}, v(\cdot)+w(\cdot)\right)-f_{k}\left(x, t, v, v_{x}^{k}, v_{x x}^{k}, v(\cdot)\right)\right] \\
\leqq \omega_{k}\left(t,\|z\|,\|w\|_{t}\right) \\
\left(\operatorname{sgn} z^{k}\right)\left[g_{k}\left(x, t, v+z, v_{\nu}^{k}, v(\cdot)+w(\cdot)\right)-g_{k}\left(x, t, v, v_{\nu}^{k}, v(\cdot)\right)\right] \\
\leqq \gamma_{k}\left(t,\|z\|,\|w\|_{t}\right) .
\end{array}\right.
$$

These inequalities are needed for $k=1,2, \cdots, m$ and for

$$
z=u-v, \quad w(\cdot)=u(\cdot)-v(\cdot) .
$$

However, since only one of the functions $u$ or $v$ is usually known, we state conditions of this kind for arbitrary $z \in \boldsymbol{R}^{m}$ and $w(\cdot) \in Z^{m}$, here and below.

The following elementary result serves as a preliminary to Theorem 1. 
THeOREm 0. Let $G_{0}$ be bounded and let $(P, R)$ satisfy (4ab), where $\omega$ and $\gamma$ are quasimonotone increasing. Suppose also that $\rho$ is an increasing solution of

$$
\rho(0)>\|u-v\|_{0}, \quad \rho_{t}>\omega(t, \rho, \rho)+\delta, \quad \rho>\gamma(t, \rho, \rho)+\varepsilon
$$

on $(0, T]$. Then

$$
\begin{aligned}
\|P u-P v\| & \leqq \delta \text { in } G \text { and }\|R u-R v\| \leqq \varepsilon \text { in } \Gamma \\
& =\|u-v\| \leqq \rho \text { in } G_{0} .
\end{aligned}
$$

5. Proof. If the conclusion fails we can find an index $k$ and a point $(\xi, \tau) \in G_{0}$ such that $\tau>0$ and

$$
\left|u^{k}(\xi, \tau)-v^{k}(\xi, \tau)\right|=\rho^{k}(\tau),
$$

while for $j=1,2, \cdots, m$

$$
\left|u^{j}(x, t)-v^{j}(x, t)\right| \leqq \rho^{j}(t) \text { for }(x, t) \in G_{0}, t \leqq \tau .
$$

We assume $u^{k}-v^{k}=+\rho^{k}$ at $(\xi, \tau)$; the discussion with - is similar. Since $\rho$ is increasing, we have

$$
\begin{array}{ll}
u^{k}(\xi, \tau)-v^{k}(\xi, \tau)=\rho^{k}(\tau), & \|u(\xi, \tau)-v(\xi, \tau)\| \leqq \rho(\tau), \\
\left|u^{k}-v^{k}\right|_{\tau}=\rho^{k}(\tau), & \|u-v\|_{\tau} \leqq \rho(\tau) .
\end{array}
$$

We consider three cases.

Case I. $(\xi, \tau)$ is a boundary point of type $I$. The relation

$$
\begin{aligned}
R_{k} u-R_{k} v & \geqq \rho^{k}(\tau)-\gamma_{k}\left(\tau,\|u-v\|,\|u-v\|_{\tau}\right) \\
& \geqq \rho^{k}(\tau)-\gamma_{k}(\tau, \rho(\tau), \rho(\tau))>\varepsilon^{k}(\tau)
\end{aligned}
$$

holds at $(\xi, \tau)$ and contradicts the hypothesis $\|R u-R v\| \leqq \varepsilon$.

Case II. $(\xi, \tau)$ is a boundary point of type II. Here we have

$$
u_{\nu}^{k} \leqq v_{\nu}^{k} \text { at }(\xi, \tau)
$$

in addition to the relations above. The monotonicity of $g_{k}$ gives

$$
R_{k} u-R_{k} v \geqq \rho_{k}-g_{k}\left(\xi, \tau, u, v_{\nu}, u(\cdot)\right)+g_{k}\left(\xi, \tau, v, v_{\nu}, v(\cdot)\right)
$$

at $(\xi, \tau)$, and a contradiction is obtained as in Case I above.

Case III. $(\xi, \tau) \in G$. Here we have

$$
u_{t}^{k}-v_{t}^{k} \geqq \rho_{t}^{k}, \quad u_{x}^{k}=v_{x}^{k}, \quad u_{x x}^{k} \leqq v_{x x}^{k}
$$

at $(\xi, \tau)$, in addition to the relations of Case I. Hence, by the 
monotonicity of $f_{k}$,

$$
\begin{aligned}
\rho_{t}^{k} \leqq & u_{t}^{k}-v_{t}^{k}=P_{k} u+f_{k}\left(\xi, \tau, u, u_{x}^{k}, u_{x x}^{k}, u(\cdot)\right) \\
& \quad-P_{k} v-f_{k}\left(\xi, \tau, v, v_{x}^{k}, v_{x x}^{k}, v(\cdot)\right) \\
\leqq & \delta^{k}+f_{k}\left(\xi, \tau, u, v_{x}^{k}, v_{x x}^{k}, u(\cdot)\right)-f_{k}\left(\xi, \tau, v, v_{x}^{k}, v_{x x}^{k}, v(\cdot)\right) \\
\leqq & \delta^{k}+\omega_{k}\left(\tau,\|u-v\|,\|u-v\|_{\tau}\right) \\
\leqq & \delta^{k}+\omega_{k}(\tau, \rho(\tau), \rho(\tau))<\rho_{t}^{k},
\end{aligned}
$$

which is a contradiction.

6. Formulation of the main theorem. We now suppose that the coordinates $x_{1}, x_{2}, \cdots, x_{l}$ are unbounded as $(x, t)$ ranges over $G$ and that the remaining coordinates are bounded. It is also assumed that the boundary operator $R$ does not depend on $u(\cdot)$, so that

$$
\left\{\begin{array}{l}
P_{k} u=u_{t}^{k}-f_{k}\left(x, t, u, u_{x}^{k}, u_{x x}^{k} \uparrow, u(\cdot)\right), \\
R_{k} u=u^{k}-g_{k}\left(x, t, u, u_{\nu}^{k} \uparrow\right) .
\end{array}\right.
$$

The argument $u_{\nu}^{k}$ is omitted at points of Type I. As continuity conditions we require

$$
\left\{\begin{array}{l}
\left(\operatorname{sgn} z^{k}\right)\left[f_{k}\left(x, t, v+z, v_{x}^{k}+p, v_{x x}^{k}+q, v(\cdot)+w(\cdot)\right)\right. \\
\left.\quad-f_{k}\left(x, t, v, v_{x}^{k}, v_{x x}^{k}, v(\cdot)\right)\right] \leqq \omega_{k}\left(t,\|z\|,\|w\|_{t}\right)+N|x||p|+N|x|^{2}|q|, \\
\left(\operatorname{sgn} z^{k}\right)\left[g_{k}\left(x, t, v+z, v_{\nu}^{k}+s\right)-g_{k}\left(x, t, v, v_{\nu}^{k}\right)\right] \leqq \gamma_{k}(t,\|z\|) \\
+N|x||s| .
\end{array}\right.
$$

In these relations $z \in \boldsymbol{R}^{m}, p \in \boldsymbol{R}^{n}, q \in S^{n}, w(\cdot) \in Z^{m}, s \in \boldsymbol{R}$ and $N$ is a large constant that does not enter the final estimate. The inequality (5b) for $f_{k}$ is required in the following two cases:

(i) $|x|>N,|p|<1 / N|x|,|q|<1 / N|x|^{2}, p_{i}=q_{i j}=0$ for $i, j>l$,

(ii) $|x| \leqq N, p=0, q=0$.

The inequality (5b) for $g_{k}$ is required in these cases:

(i) $|x|>N,|s|<1 / N|x|$,

(ii) $|x| \leqq N, s=0$.

At points of Type I, the condition involving $s$ is vacuous.

Finally, we assume that

$$
\left\{\begin{array}{l}
\omega_{k}(t, y+z, y+\bar{z})-\omega_{k}(t, y, y) \leqq K(|z|+|\bar{z}|) \text { for } z \geqq 0, \bar{z} \geqq 0, \\
\gamma_{k}(t, y+z)-\gamma_{k}(t, y) \leqq \sum_{j=1}^{m} K_{k j} z_{j} \text { for } z \geqq 0,
\end{array}\right.
$$

where $K, K_{i j}$ are constants satisfying $K \geqq 0, K_{i j} \geqq 0$ for $i \neq j$, $\sum_{j=1}^{m} K_{i j}<1$.

THEOREM 1. Let $(P, R)$ satisfy (5abc) where $\omega$ and $\gamma$ are quasimonotone increasing. Suppose also that $\rho$ is an increasing 
solution of

$$
\rho(0) \geqq\|u-v\|_{0}, \quad \rho_{t} \geqq \omega(t, \rho, \rho)+\delta, \quad \rho \geqq \gamma(t, \rho)+\varepsilon
$$

on $(0, T]$. Then

$$
\begin{gathered}
\|P u-P v\| \leqq \delta \text { in } G, \quad\|R u-R v\| \leqq \varepsilon \text { in } \Gamma, \sup _{G_{0}}\|u-v\|<\infty \\
\Longrightarrow\|u-v\| \leqq \rho \text { in } G_{0} .
\end{gathered}
$$

The choice $v=0, \rho=z$ in Theorem 1 gives the following:

Corollary 1. Let $P$ be as in Theorem 1, with $v=0$ in (5b), and let $u$ be a bounded solution of $P u=P 0$. Suppose $z \in \boldsymbol{R}_{+}^{m}$ is such that $\omega(t, z, z) \leqq 0$ for $0<t \leqq T$. Then $\|u\|$ satisfies the maximum principle relative to $z$; that is, $\|u(x, t)\| \leqq z$ in $\Gamma$ implies $\|u(x, t)\| \leqq z$ in $G$.

This follows from Theorem 1 by taking $g=\gamma=\delta=0, \rho=\varepsilon=z$.

If $\omega(t, y, z)=L(t) y+K(t) z$ is linear the set of vectors $z$ in Corollary 1 is defined by

$$
L(t) z+K(t) z \leqq 0 \leqq z, \quad 0<t \leqq T,
$$

and hence is a convex cone. Further discussion of the linear case is given in the following section.

\section{Remarks and generalizations.}

REMARK 1. The hypothesis (5c) with regard to $\gamma$ can be relaxed as follows. It suffices to assume that

(i ) $\gamma(t, y+z)-\gamma(t, y) \leqq M(t) z$ for $z \geqq 0$, where $M(t)$ is a matrix-valued function continuous in $[0, T]$ such that

(ii) $\sigma(t)>M(t) \sigma(t)$ has a solution $\sigma(t)>0$ in $[0, T]$.

First we note that this assumption is weaker than the original one. Indeed, if $M(t)=M=\left(K_{i j}\right)$, where the $K_{i j}$ are the constants in (5c), then $\sigma(t)=(1,1, \cdots, 1)$ satisfies (ii). Second, we may assume that the function $\sigma$ in (ii) is smooth. For, if (ii) holds, then the HeineBorel theorem gives a finite set of relatively open intervals $I_{i}$ covering $[0, T]$ and a set of constant vectors $\xi_{i}$ such that $\xi_{i}>0, \xi_{i}>M(t) \xi_{2}$ for $t \in I_{i}$. If $\mu_{i}$ are smooth functions $\boldsymbol{R} \rightarrow \boldsymbol{R}$ such that

$$
\mu_{i}(t)>0 \text { for } t \in I_{i}, \mu_{i}(t)=0 \text { for } t \in[0, T]-I_{i},
$$

then the function $\bar{\sigma}(t)=\sum \xi_{i} \mu_{i}(t)$ is smooth and satisfies (ii).

REMARK 2. The first part of hypothesis (5c) is needed only 
when $y=\sigma(t)$ and $z \| \sigma(t)$, where $\sigma:[0, T] \rightarrow R^{m}$ is any continuous function satisfying inf $\sigma_{t}>-\infty$ as well as (i), (ii) above. Here $z \| \sigma(t)$ means $z=\lambda(t) \sigma(t), \lambda \geqq 0$. In any case (5c) is needed only for $|z|,|\bar{z}| \leqq \sup |u-v|$ and the constants can depend on $(u, v)$. They do not enter into the statement of Theorem 1.

Remarks 1 and 2 are most easily understood when $\omega$ and $\gamma$ are linear. Let

$$
\omega(t, y, z)=L(t) y+K(t) z, \quad \gamma(t, y)=M(t) y
$$

where $y \in \boldsymbol{R}^{m}, z \in \boldsymbol{R}^{m}$ and where $L, K, M$ are $m$ by $m$ matrix-valued functions of $t$. The conditions for $\rho$ now take the form

$$
\rho_{t} \geqq L(t) \rho+K(t) \rho+\delta, \quad \rho \geqq M(t) \rho+\varepsilon
$$

and the quasimonotonicity of $\omega$ and $\gamma$ is equivalent to

$$
L_{i j}(t) \geqq 0, \quad K_{i j}(t) \geqq 0, \quad M_{i j}(t) \geqq 0 \text { for } i \neq j, \quad 0 \leqq t \leqq T .
$$

The hypothesis (5c) as restricted by Remarks 1 and 2 becomes

$$
L(t) \sigma(t) \leqq \text { const. , } \quad K(t) \leqq \text { const. , } M(t) \sigma(t)<\sigma(t) .
$$

Here the first and third inequalities pertain to the ordering in $\boldsymbol{R}^{m}$ and the second means that each element $K_{i j}(t)$ is bounded above. Since the diagonal elements of $L$ and $M$ can be negative, these conditions are much weaker than corresponding original conditions

$$
\sum_{j} L_{i j}(t) z_{j} \leqq \text { const. }|z| \text { for } z \geqq 0, \quad M_{i j}(t) \leqq K_{i j}, \quad \sum_{j} K_{i j}<1 .
$$

When $L$ is bounded above, any $\sigma$ can be used with $L$, and hence the $\sigma$ from Remark 1 can be. Since the $k$ th boundary operator $R_{k}$ must actually involve $u^{k}$, it does not appear that the condition on $M$ given in Remark 1 can be significantly weakened.

If $M$ is a diagonal matrix with diagonal elements bounded above by $K_{0}<1$, the sole condition on the continuous function $\sigma$ (other than $\sigma>0$, inf $\left.\sigma_{t}>-\infty\right)$ is $L(t) \sigma(t) \leqq$ const. Such a condition can hold when the elements of $L$ are severely unbounded, as is evident. It should be observed in this connection that $\sigma, K$ and $K_{0}$ do not enter into the conclusion of Theorem 1. Only their existence is important, not their values.

REMARK 3. The hypothesis $\|u-v\|_{0} \leqq \rho(0)$ can be replaced by the boundary condition

$$
\|R u-R v\| \leqq \varepsilon(0), \quad t \leqq 0,
$$

provided the following conditions hold: $\rho(0) \geqq \gamma[t, \rho(0)]+\varepsilon(0)$ and 
(iii) $M(t)=M(0)$ for $t \leqq 0$,

(iv) $\xi \in \boldsymbol{R}^{m}, \xi>M(0) \xi \Rightarrow \xi>0$.

To see this, note that the boundary condition $\|R u-R v\| \leqq \varepsilon(0)$ for $t \leqq 0$ gives

$$
\|u-v\| \leqq \varepsilon(0)+M(0)\|u-v\| \quad(t \leqq 0) .
$$

On the other hand the comparison function $\psi$ used in the proof of Theorem 1 satisfies

$$
(I-M) \psi \geqq \lambda_{i}(I-M) \sigma+(I-M) \rho>\varepsilon(0) \quad(t \leqq 0) .
$$

Putting these two results together gives $(I-M)(\psi-\|u-v\|)>0$ for $t \leqq 0$, and Property (iv) ensures

$$
\|u(x, t)-v(x, t)\|<\psi(x, t), \quad t \leqq 0 .
$$

In the proof of Theorem 1, which is given below, it will be seen that the only use of the condition $\rho(0) \geqq\|u-v\|_{0}$ is to establish (*). This completes the proof of Remark 3 .

In view of the above remarks, Theorem 1 implies the theorem for the linear case which was stated without proof at the end of [9].

REMARK 4. In most cases of interest a term $\|u\|_{t}$ does not occur in the original statement of the problem, but arises by assessment of a Volterra-type functional. In such cases one would naturally expect $K_{i j} \geqq 0$ for $i=j$ as well as for $i \neq j$. Even if $\|u\|_{t}$ does occur in the original problem, the comparison function $v$ must be severely restricted, in general, before $K_{i i}<0$ can be allowed. As an illustration consider

$$
P u=u_{t}-\Delta u+\sup _{|x|<\infty} u(x, t), \quad m=1 .
$$

Here we can take $K=-1$ provided $v$ is a function of $t$ alone. If the sup were replaced by $\sup u(x, \tau)$ for $\tau \leqq t$, or by a sup with respect to both $x$ and $\tau$, we could take $K=-1$ when $v$ is constant.

Equations involving maximum operators such as the above do arise in the theory of dynamic programming and elsewhere. However, we have allowed $\omega(t, y, z)$ to be merely quasimonotonic in $z$, rather than monotonic, not with a view to any esoteric application, but to show the parallelism between the dependence on $u$ and that on $u(\cdot)$.

REMark 5. Let $|\dot{\phi}|_{t}^{*}$ and $\|w\|_{t}^{*}$ be defined as in Definition 2 in $\S 2$, but with $\tau \leqq t$ replaced by $\tau=t$ (i.e., the supremum is taken 
only over $x$ with $t$ fixed). If the estimate (5b) for $f$ holds with $\|w\|_{t}$ replaced by $\|w\|_{t}^{*}$, then the assumption in Theorem 1 that $\rho$ is increasing can be dropped.

This remark applies, e.g., when the functional in $P$ is of the form $u(x+\xi(x, t), t)$, that is, when we have no delay in $t$, but a deviating argument in $x$. Further discussion is given in $\S 10$.

8. Proof of Theorem 1. The side conditions associated with Theorem 1 involve an index $l$ such that $x_{1}, x_{2}, \cdots, x_{l}$ are unbounded in $G_{0}$ and the remaining coordinates are bounded. We define

$$
\bar{x}=\left(x_{1}, x_{2}, \cdots, x_{l}\right)
$$

and increase $N$, if necessary, so that $N>1$ and also

$$
(x, t) \in G_{0}, \quad|x| \geqq N \Longrightarrow|\bar{x}| \leqq|x| \leqq 2|\bar{x}| .
$$

We shall prove the theorem under the weaker assumptions outlined in Remarks 1 and 2 of the preceding section.

Let $\sigma$ be a smooth function satisfying (ii) in Remark 1, and let $\sigma_{0}, \sigma_{1}$ be constants such that

$$
0<\sigma_{1}<1<\sigma_{0}, \sigma_{0} \sigma^{k}(t)>|\sigma(t)| \text { and }(\sigma(t)-M(t) \sigma(t))^{k}>\sigma_{1}
$$

for $0 \leqq t \leqq T, k=1, \cdots, m$. Let

$$
\mu=\sigma_{0} K+(2+4 \sqrt{n}) N, \quad \beta=\sigma_{0} K+\sup |u-v| .
$$

By replacing $\sigma$ by the function $c e^{c t} \sigma(t)$, where $c$ is a large constant, we can assume that $\sigma$ satisfies

$$
\sigma_{t}>\mu \sigma \text { and } \sigma^{k}>1(k=1, \cdots, m) \text { in }[0, T] .
$$

Note that this change does not affect the properties (6) of $\sigma$. It will be proved by induction that

$$
\|u(x, t)-v(x, t)\| \leqq \rho(t)+\lambda_{i}(t) \sigma(t) \text { for } i=0,1,2, \cdots,
$$

where

$$
\lambda_{i}(t)=\beta^{i+1}(t+1)^{i} / i ! \quad(i=0,1,2, \cdots) .
$$

Obviously, the conclusion of Theorem 1 follows from (9) for $i \rightarrow \infty$. Since $\rho^{k}>0, \sigma^{k}>1$ and $\beta>|u-v|$, inequality (9) holds for $i=0$. Let us assume therefore that (9) holds for the index $i-1$. In order to prove (9) for the index $i$, we shall establish the estimate

$$
\|u(x, t)-v(x, t)\| \leqq \rho(t)+\left(\alpha h(x)+\lambda_{i}(t)\right) \sigma(t)
$$

for all $\alpha$ satisfying 


$$
0<\alpha<(4 N \sqrt{n} \sup |\sigma|)^{-1}
$$

where

$$
\begin{gathered}
h(x)= \begin{cases}0 & \text { for }|x| \leqq N \\
\alpha_{0}+\log |\bar{x}| & \text { for }|x|>N\end{cases} \\
\alpha_{0}=\frac{2 N}{\sigma_{1}} \sup \mid \sigma_{\mid}+1
\end{gathered}
$$

In what follows, we set

$$
w=u-v, \quad \psi(x, t)=\rho(t)+\left(\alpha h(x)+\lambda_{i}(t)\right) \sigma(t),
$$

and we define

$$
\rho(t)=\rho(0), \quad \sigma(t)=\sigma(0), \quad \lambda_{i}(t)=\lambda_{i}(0) \text { for } t<0 .
$$

The conclusion $\|w(x, t)\|<\psi(x, t)$ holds for $t \leqq 0$ by virtue of the hypothesis on $\rho(0)$ together with the fact that $\sigma(0)>0$. If this conclusion does not hold for all $(x, t) \in G_{0}$ then we can find a point $(\xi, \tau) \in G_{0}$ and an index $k$ such that $\tau>0$ and

$$
\left|w^{k}(\xi, \tau)\right|=\psi^{k}(\xi, \tau), \quad\|w(x, t)\| \leqq \psi(x, t) \text { for } t \leqq \tau .
$$

A point of this kind is called a Nagumo point. We assume $w^{k}=+\psi^{k}$ at the Nagumo point; discussion with - is similar. Thus, the basic conditions are $\tau>0$ and

$$
w^{k}(\xi, \tau)=\psi^{k}(\xi, \tau), \quad\|w(x, t)\| \leqq \psi(x, t) \text { for } t<\tau .
$$

The possible locations of $(\xi, \tau)$ lead to the cases considered below.

Case I. Boundary point of Type I, $|\xi|>N$. Using first the quasimonotonicity of $\gamma$ and then the hypothesis on $\rho$, we get

$$
\begin{aligned}
R_{k} u-R_{k} v & \geqq \psi^{k}(\xi, \tau)-\gamma_{k}(\tau, \psi(\xi, \tau)) \\
& \geqq \varepsilon^{k}(\tau)+\lambda \sigma^{k}(\tau)+\gamma_{k}(\tau, \rho(\tau))-\gamma_{k}(\tau, \rho(\tau)+\lambda \sigma(\tau)),
\end{aligned}
$$

where we have introduced the abbreviation

$$
\lambda=\alpha h(\xi)+\lambda_{i}(\tau)
$$

We apply (5c) with $y=\rho(\tau)$ and $z=\lambda \sigma(\tau)>0$. Thus, we get a contradiction,

$$
R_{k} u-R_{k} v \geqq \varepsilon^{k}(\tau)+\lambda \sigma^{k}(\tau)-(M(\tau) \lambda \sigma(\tau))^{k}>\varepsilon^{k}(\tau) .
$$

Case II. Boundary point of Type II, $|\xi|>N$. Here we have, besides the conditions used in Case I, the condition $u_{\nu}^{k} \leqq v_{\nu}^{k}+\alpha h_{\nu} \sigma^{k}(\tau)$. 
Since $\left|h_{\nu}\right| \leqq\left|h_{x}\right| \leqq 2 /|x|$ because of $|x| \leqq 2|\bar{x}|$, the smallness of $\alpha$ ensures $|s| \leqq 1 / N|x|$ for $s=\alpha h_{\nu} \sigma^{k}(\tau)$. Upon using first the monotonicity of $g$, then the inequality involving $\gamma$, and finally the results noted in Case I, we see that

$$
R_{k} u-R_{k} v \geqq \varepsilon^{k}(\tau)+\lambda(\sigma(\tau)-M(\tau) \sigma(\tau))^{k}-2 \alpha N \sigma^{k}(\tau) .
$$

Since $\lambda \geqq \alpha \alpha_{0}$ and $\alpha_{0} \sigma_{1}>2 N \sup |\sigma|$, a contradiction is obtained again.

Case III. Interior point, $|\xi|>N$. Here we have (11) and also

$$
w_{t}^{k} \geqq \psi_{t}^{k}, \quad u_{x}^{k}-v_{x}^{k}=\alpha h_{x} \sigma^{k}, \quad u_{x x}^{k}-v_{x x}^{k} \leqq \alpha h_{x x} \sigma^{k} \text { at }(\xi, \tau) \text {. }
$$

Since $\left|h_{x}\right| \leqq 2 /|x|$ and $\left|h_{x x}\right| \leqq 4 \sqrt{n} /|x|^{2}$, the smallness of $\alpha$ assures the side conditions (i) following (5b). Using first the monotony with respect to $u_{x x}$ given by (5a), and then the continuity hypothesis (5b), we get

$$
P_{k} u-P_{k} v \geqq \psi_{t}^{k}(\xi, \tau)-\omega_{k}\left(\tau,\|w(\xi, \tau)\|,\|w\|_{\tau}\right)-\alpha(2+4 \sqrt{n}) N \sigma^{k}(\tau) .
$$

By (11) and the quasimonotonicity of $\omega$ we can replace $\|w(\xi, \tau)\|$ on the right by $\psi(\xi, \tau)$. To effect a similar substitution for $\|w\|_{t}$ let us define $s \in \boldsymbol{R}^{m}$ by

$$
\left|w^{j}\right|_{t}=\rho^{j}(t)+s^{j} \text { if }\left|w^{j}\right|_{t} \geqq \rho^{j}(t),
$$

otherwise $s^{j}=0$. Then we can replace $\|w\|_{t}$ by $\rho(t)+s$ to get

$$
P_{k} u-P_{k} v \geqq \psi_{t}^{k}(\xi, \tau)-\omega_{k}(\tau, \psi(\xi, \tau), \rho(\tau)+s)-\alpha(2+4 \sqrt{n}) N \sigma^{k}(\tau) .
$$

Upon recalling that

$$
\psi(x, t)=\alpha h(x) \sigma(t)+\lambda_{i}(t) \sigma(t)+\rho(t)
$$

and that $\sigma_{t} \geqq \mu \sigma$ by (8), we see that $\psi$ satisfies the differential inequality

$$
\psi_{t}>\left(\alpha h+\lambda_{i}\right) \mu \sigma+\beta \lambda_{i-1} \sigma+\omega(t, \rho, \rho)+\delta .
$$

On the other hand with $\lambda=\alpha h(\xi)+\lambda_{i}(\tau)$ the hypothesis (5c) gives

$$
\begin{gathered}
\omega_{k}(\tau, \rho(\tau)+\lambda \sigma(\tau), \rho(\tau)+s)-\omega_{k}(\tau, \rho(\tau), \rho(\tau)) \leqq K(\lambda|\sigma(\tau)|+|s|) \\
\leqq K\left(\lambda \sigma_{0} \sigma^{k}(\tau)+\lambda_{i-1}(\tau) \sigma_{0} \sigma^{k}(\tau)\right)
\end{gathered}
$$

In the last step we used the fact that $|\sigma| \leqq \sigma_{0} \sigma^{k}$ by (6), and also the induction hypothesis (9) for the index $i-1$. The latter gives $|s| \leqq \lambda_{i-1}(\tau)|\sigma(\tau)|$. Because of the inequalities satisfied by $\alpha_{0}, \beta$ and $\mu$, the two inequalities above lead to a contradiction: 


$$
P_{k} u-P_{k} v>\delta^{k}+\left(\lambda \mu+\beta \lambda_{i-1}-K \sigma_{0}\left(\lambda+\lambda_{i-1}\right)-\alpha(2+4 \sqrt{n}) N\right) \sigma^{k}>\delta^{k}
$$

at $(\xi, \tau)$.

Case $I V .|\xi| \leqq N$. If $\|w\| \leqq \psi$ fails, it has been seen that there is no Nagumo point in $|x|>N$, and hence there must be one in $|x| \leqq N$. This is true for every $\alpha>0$. In general, both the point and the distinguished index $k$ might be expected to depend on $\alpha$; thus, $(\xi, \tau)=\left(\xi_{\alpha}, \tau_{\alpha}\right), k=k_{\alpha}$. However, since $h(x)=0$ for $|x| \leqq N$, it is not hard to show that one and the same point $(\xi, \tau)$ and index $k$ can be used for all $\alpha$ as $\alpha \rightarrow 0+$. This point is also a Nagumo point when $\alpha=0$, and a contradiction is obtained as in the proof of Theorem 0 in $\S 5$.

The result of the foregoing analysis is that (10) holds for $\alpha=0$, hence (9) holds for the index $i$, and the proof by induction is completed.

9. Remarks on strongly coupled systems. In this section and the next we give examples of the foregoing theory, making special choices of the functional implied in $u(\cdot)$.

Partial derivatives are denoted by the usual indicial notation,

$$
D^{\alpha}=\partial^{|\alpha|} / \partial x_{1}^{\alpha_{1}} \partial x_{2}^{\alpha_{2}} \cdots \partial x_{n}^{\alpha_{n}}, \quad|\alpha|=\alpha_{1}+\alpha_{2}+\cdots+\alpha_{n} .
$$

If $0<\theta<\pi / 2$ and $h>0$, a cone $C(\theta, h)$ with vertex at 0 , vertex angle $2 \theta$ and height $h$ is the set of all $x \in R^{n}$ satisfying

$$
e_{0} x \geqq|x| \cos \theta, \quad|x| \leqq h .
$$

Here $e_{0}$ is a unit vector defining the axis of the cone and $e_{0} x$ denotes the inner product. When $n=1$ we agree that $C(\theta, h)$ denotes a line segment of length $h$. The set $\Omega \subset R^{n}$ belongs to the class $K(\theta, h)$ if for each $x \in \Omega$ there exists a cone $C(\theta, h)$ such that $x+C(\theta, h) \subset \Omega$.

If $\Omega \subset \boldsymbol{R}^{n}$ is an open set, the class $C^{d}\left(\Omega, \boldsymbol{R}^{m}\right)$ is the class of functions $\Omega \rightarrow \boldsymbol{R}^{m}$ such that all derivatives of order $\leqq d$ are continuous. The class $C_{\phi}^{d}\left(\Omega, \boldsymbol{R}^{m}\right)$ is the subclass of functions in $C^{d}\left(\Omega, \boldsymbol{R}^{m}\right)$ for which the derivatives of order $d$ satisfy

$$
\left|D^{\beta} u(x)-D^{\beta} u(y)\right| \leqq \phi(|x-y|), \quad|\beta|=d,
$$

where $\phi$ is a modulus of continuity; that is, $\phi$ is continuous and $\phi(0)=0$. In our applications we shall have $\Omega \in K(\theta, h)$ and (12) is required only for $(x, y)$ such that

$$
|x-y| \leqq h, \quad \lambda x+(1-\lambda) y \in \Omega, \quad 0 \leqq \lambda \leqq 1 .
$$

Hence, by the mean-value theorem, the condition holds with $\phi(s)=$ 
(const)s if all derivatives of order $d+1$ are bounded.

The following lemma is proved in [11]:

LEMMA 1. Let $\Omega \subset R^{n}$ be an open set of class $K(\theta, h)$, bounded or unbounded, and let $w \in C_{\phi}^{d}\left(\Omega, R^{m}\right)$ for some modulus of continuity $\phi$. Let

$$
W_{k}=\sup \left\{\left|D^{\alpha} w(x)\right|:|\alpha|=k, x \in \Omega\right\} \quad(k=0,1, \cdots, d)
$$

where $W_{0}<\infty$. Then $W_{k}<\infty$, and there exists a modulus of continuity $\Phi$, depending only on $(\theta, h, d, m, \phi)$, such that

$$
W_{0}+W_{1}+\cdots+W_{d} \leqq \Phi\left(W_{0}\right) \text {. }
$$

Actually the result is deduced in [11] from a hypothesis which is much weaker than $w \in C_{\phi}^{d}\left(\Omega, R^{m}\right)$. However it follows from the conclusion that in fact $w \in C_{\phi}^{d}\left(\Omega, R^{m}\right)$ for some $\phi$ and hence, the above formulation has been preferred here.

These considerations are extended to functions $u(x, t)$ defined on the region $G$ by means of the following definition:

Definition 4. Let $G_{t}$ denote the nonempty cross sections

$$
G_{t}=\left\{x \in \boldsymbol{R}^{n}:(x, t) \in G\right\} .
$$

Then $G$ belongs to the class $K(\theta, h)$ if each $G_{t}$ does so, with $(\theta, h)$ independent of $t$. The function $w: G \rightarrow R^{m}$ belongs to $C_{\phi}^{d}\left(G, R^{m}\right)$ if $w(t, \cdot)$ belongs to $C_{\phi}^{d}\left(G_{t}, R^{m}\right)$, where the modulus of continuity $\phi$ is independent of $t$.

It is convenient to denote by $D u$ the vector of $m\left(1+n+\cdots+n^{d}\right)$ components which contains all $x$-derivatives $D^{\alpha} u,|\alpha| \leqq d$. Thus, $D$ is of order $d$. We consider the operators

$$
\begin{cases}P_{k} u=u_{t}^{k}-f_{k}\left(x, t, u, u_{x}^{k}, u_{x x}^{k} \uparrow, D u\right) & \text { in } G \\ R_{k} u=u^{k}-g\left(x, t, u_{\nu}^{k} \uparrow\right) & \text { in } \Gamma=\bar{G}-G,\end{cases}
$$

where $D u$ is an abbreviation for $(D u)(x, t)$ and where

$$
\begin{aligned}
\left(\operatorname{sgn} z^{k}\right)[ & f_{k}\left(x, t, v+z, v_{x}, v_{x x}, D v+s\right) \\
& \left.\quad-f_{k}\left(x, t, v, v_{x}, v_{x x}, D v\right)\right] \leqq \omega(t,|z|,|s|)
\end{aligned}
$$

for $k=1,2, \cdots, m$. Here $s=\left(s_{\alpha}\right),|\alpha| \leqq d$, and the function $\omega:[0, T] \times$ $\boldsymbol{R}^{2} \rightarrow \boldsymbol{R}$ satisfies

$\omega$ is continuous, increasing in the last argument, and

$$
\omega(0,0,0)=0 \text {. }
$$


THEOREM 2. Let $(P, R)$ satisfy (13abc) in a bounded region $G \subset$ $K(\theta, h)$ and let $(u, v)$ belong to $C_{\phi}^{d}\left(G, \boldsymbol{R}^{m}\right)$ with $d \geqq 1$. Then the conditions

$$
\begin{gathered}
|P u-P v|=o(1) \text { and }|R u-R v|=o(t) \text { as } t \longrightarrow 0+ \\
\text { uniformly in } x
\end{gathered}
$$

imply

$$
\begin{aligned}
& |D u-D v|=o(1) \text { and }|u-v|=o(t) \text { as } t \longrightarrow 0+ \\
& \text { uniformly in } x .
\end{aligned}
$$

In particular, if either of the forward derivatives $\partial u / \partial t$ or $\partial v / \partial t$ exists at $(x, 0)$ then the other exists and has the same value.

Before giving the proof we illustrate Theorem 2 by two examples.

ExAmple 1. Let $n=1, x^{1}=x$, and let $G$ be defined by $t>x^{2}$. Consider the problem

$$
2 u_{t}^{1}=u_{x x}^{1}-2 u_{x x}^{2}, \quad 2 u_{t}^{2}=u_{x x}^{2}-2 u_{x x}^{1}, \quad(x, t) \in G,
$$

subject to $u^{1}=u^{2}=0$ on $\Gamma$. This problem has the two solutions

$$
u=\left(u^{1}, u^{2}\right)=\left(t-x^{2}, t-x^{2}\right) ; \quad v=\left(v^{1}, v^{2}\right)=(0,0),
$$

which do not satisfy the conclusion of Theorem 2. Nevertheless, all the hypotheses of Theorem 2, except one, are satisfied with a great deal to spare, and both $u$ and $v$ are of class $C^{\infty}$. The only condition which fails is $G \in K(\theta, h)$. To be sure, each cross section $G_{t}$ satisfies $G_{t} \in K\left(\theta, t^{1 / 2}\right)$; but the parameter $h=t^{1 / 2}$ tends to 0 with $t$, and hence the condition does not hold uniformly in the sense required by Definition 4. This uniformity is therefore essential for the truth of Theorem 2, even when $\Gamma$ is smooth.

EXAMPLE 2. If the hypothesis of Theorem 2 is strengthened to $P u=P v$ and $R u=R v$, and $\omega$ is linear, one might expect that the conclusion could be strengthened to $u=v$. However, this is not the case. Using the notation of Example 1, let $G$ be bounded by the three lines $x+t=0, t=0, x=1$, and consider the system

$$
\left\{\begin{array}{l}
5 u_{t}^{1}=u_{x x}^{1}-(1-x) u_{x x}^{2}-3 u_{x}^{1} \\
5 u_{t}^{2}=u_{x x}^{2}-(1-x) u_{x x}^{1}-3 u_{x}^{2}
\end{array} \quad(x, t) \in G\right.
$$

subject to $u=0$ on $\Gamma$. This problem has the solution

$$
u=\left(x^{4} t+x^{5}, x^{4} t+x^{5}\right) \quad(x \leqq 0) ; \quad u=(0,0) \quad(x \geqq 0)
$$


as well as the null solution $v=(0,0)$. All the hypotheses of Theorem 2 hold and, in agreement with the conclusion, $\partial u / \partial t=\partial v / \partial t$ for $t=0,0<x<1$. Nevertheless, $u \neq v$.

The proof of Theorem 2 is simple. By Lemma 1 we can find a modulus of continuity $\Phi(s)$ such that $w=u-v$ satisfies

$$
|D w| \leqq \Phi\left(\sup _{G_{t}}|w|\right)
$$

The condition for $\rho$ in Theorem 0 is therefore

$$
\rho(0)>0, \quad \rho_{t}>\omega(t, \rho, \Phi(\rho))+\lambda(t), \quad \rho>t \lambda(t),
$$

where $\lambda$ is continuous and $\lambda(0)=0$. These can be regarded as scalar equations with $\rho^{k}=\rho, \omega^{k}=\omega$ for $k=1, \cdots, m$. Given $\lambda \epsilon$ $(0,1 / 2)$, there exists $\mu>0$ such that

$$
0<t<\rho, 0<\rho<\mu \Longrightarrow \lambda(t)+\omega(t, \rho, \Phi(\rho))<\lambda .
$$

Hence we may take $\rho(t)=\lambda t+\eta, 0<\eta<\mu / 2$, and we obtain $|u-v| \leqq$ $\lambda t+\eta$ for $0 \leqq t \leqq \mu$. Letting $\eta \rightarrow 0$, the result follows.

By using the full force of Theorem 1, one can readily extend Theorem 2 to unbounded regions and to strongly coupled systems containing functionals. Also one can obtain more detailed estimates by means of the inequalities

$$
W_{k} \leqq \text { (const) } W_{0}^{1-k / d}\left[\max \left(W_{0}, W_{d}\right)\right]^{k / d}, \quad k=0,1, \cdots, d
$$

which are established for functions $w \in C^{d}\left(\Omega, R^{m}\right)$ on regions $\Omega \in$ $K(\theta, h)$ in [11]. For suitably restricted polynomials $H(D w)$ in the elements of $D w$, the inequalities (15) give an estimate of form

$$
|H(D w)| \leqq(\text { const })(\sup |w|)^{p}, \quad p \geqq 1,
$$

and this can be used to establish uniqueness, stability, and asymptotic stability for the null solutions of certain rather broad classes of strongly coupled systems. Details of these developments are not difficult and are omitted.

In conclusion, we mention that the idea of using Kolmogorovtype inequalities in the study of strongly coupled systems is due to Nickel $[4,5]$, and the fact that these results can be subsumed under a general theory of parabolic equations with functionals was pointed out by the authors $[9,10]$. An existence theory within the context of Nickel's ideas is given in [8].

10. Systems with limited memory. The reason for requiring $\rho$ to be increasing in Theorem 1 is to ensure $\|\rho\|_{t}=\|\rho(t)\|$, so that $\rho(t)$ gives an estimate not only for $w(x, t)$ at the Nagumo point, 
but also for $\|w\|_{t}$. The assumption of monotonicity does no great harm on a finite interval $[0, T]$, and, if the differential equation for $\rho$ would allow a decreasing $\rho$, we could generally take $\rho=$ constant. But on an infinite interval $[0, \infty)$ the monotonicity of $\rho$ is a serious restriction. The trouble is that one would like to establish asymptotic stability,

$$
\lim _{t \rightarrow \infty}|u(x, t)-v(x, t)|=0 \text { uniformly in } x,
$$

and this requires $\lim \rho(t)=0$.

To deal with this problem let $\mu$ be a specified function $\boldsymbol{R} \rightarrow \boldsymbol{R}$ satisfying $\mu(t) \leqq t$. Without bothering to introduce a new notation, we alter the definition of $|\phi|_{t}$ in Definition 2 as follows:

$$
|\phi|_{t}=\sup \left\{|\phi(\xi, \tau)|:(\xi, \tau) \in G_{0}, \mu(t) \leqq \tau \leqq t\right\} .
$$

This gives a corresponding value, depending on $\mu$, for

$$
\|w\|_{t}=\left(\left|w^{1}\right|_{t},\left|w^{2}\right|_{t}, \cdots,\left|w^{m}\right|_{t}\right) .
$$

Roughly speaking, an expression which can be assessed by $\|w\|_{t}$ cannot involve values of $w$ at times prior to $\mu(t)$. Such an expression can be thought to be of limited memory; a measure of the memory at time $t$ is given by $t-\mu(t)$. For example, the operator $w(x, t) \rightarrow w(x, t-1)$ has memory 1 . The operator $w(x, t) \rightarrow w(2 x-t, t)$ has memory 0 , as does also the operator $w \rightarrow D w$ introduced in the foregoing discussion. Operators given by integration over the subset of points $(\xi, \tau) \in G_{0}$ for which $\mu(t) \leqq \tau \leqq t$ are of memory $t-\mu(t)$.

When the foregoing theory is developed in this setting, the hypothesis in Theorem 1 that $\rho$ is increasing can be replaced by a requirement that $\omega(t, y, z \uparrow)$ is increasing and that

$$
\rho_{t} \geqq \omega(t, \rho(t), \tilde{\rho}(t)), \quad \tilde{\rho}^{j}(t)=\max \left\{\rho^{j}(t): \mu(t) \leqq \tau \leqq t\right\} .
$$

In particular, if $\omega(t, y, z \uparrow)$ is increasing, and also $\rho$ is decreasing, it suffices to have

$$
\rho_{t} \geqq \omega(t, \rho(\tau), \rho(\mu(t))) .
$$

Thus, the study of asymptotic stability leads to a specific class of delay differential equations.

As a simple illustration, let $a_{k}, \alpha$ and $\beta$ be given functions of type $\boldsymbol{R}^{n+1} \rightarrow \boldsymbol{R}, \boldsymbol{R}^{n+1} \rightarrow \boldsymbol{R}^{n}$, and $\boldsymbol{R}^{n+1} \rightarrow \boldsymbol{R}$, respectively, and consider

$$
\left\{\begin{array}{l}
P_{k} u=u_{t}^{k}+a_{k}(x, t) u^{k}-f_{k}\left(x, t, u_{x}^{k}, u_{x x}^{k} \uparrow, u^{*}\right) \\
u *(x, t)=u(\alpha(x, t), \beta(x, t)), \quad \mu(t) \leqq \beta(x, t) \leqq t
\end{array}\right.
$$


Thus, $u \rightarrow u^{*}$ is a Volterra displacement operator with memory $t-\mu(t)$. As continuity condition we assume that there exist constants $A$ and $B$ and an increasing function $N: \boldsymbol{R} \rightarrow \boldsymbol{R}$ such that

$$
\left\{\begin{array}{l}
\alpha_{k}(x, t) \geqq A, \quad A>B \geqq 0, \\
\left|f_{k}\left(x, t, v_{x}^{k}+p, v_{x x}^{k}+q, v^{*}+s\right)-f_{k}\left(x, t, v_{x}, v_{x x}, v^{*}\right)\right| \\
\leqq N(t)|x||p|+N(t)|x|^{2}|q|+B \max _{j}\left|s^{j}\right|
\end{array}\right.
$$

for $(x, t) \in G$ and $k=1,2, \cdots, m$. In each strip $0<t \leqq T$ this is subject to side conditions for $|x|>N(T)$ and $|x| \leqq N(T)$ similar to those in Theorem 1.

THeOREM 3. In the region $G=\boldsymbol{R}^{n} \times(0, \infty)$ let $(16 \mathrm{ab})$ hold and suppose also:

(i ) $|u(x, t)-v(x, t)|$ is bounded in each strip $0 \leqq t \leqq T$,

(ii) $\lim \mu(t)=\infty$ as $t \rightarrow \infty$.

Then $P u=P v$ implies $\lim _{t \rightarrow \infty}|u(x, t)-v(x, t)|=0$ uniformly in $x$.

For proof, let $w=u-v$. To get a useful assessment for $\left\|w^{*}\right\|$ we ought to choose $t_{0}$ so that $\mu(t) \geqq 0$ for $t \geqq t_{0}$ and work in the region $t \geqq t_{0}$. However, since the main hypotheses are invariant under a translation of $t$, we shall use Theorem 1 as it stands. The conditions for $\rho$ are satisfied if $\rho$ is a decreasing solution of

$$
\rho_{t}^{k} \geqq-A \rho^{k}(t)+B \max _{j} \rho^{j}(\mu(t)), \quad \rho(0) \geqq\|w(x, 0)\| .
$$

This can be solved by setting $\rho=\lambda(\sigma, \sigma, \cdots, \sigma)$ where $\lambda$ is a sufficiently large constant and where $\sigma$ is a decreasing solution of the scalar equation

$$
\sigma_{t} \geqq-A \sigma(t)+B \sigma(\mu(t)), \quad \sigma(0)>0, \quad \sigma(t) \geqq 0 .
$$

There is no difficulty in finding particular solutions of form $e^{-\alpha t}$, $(t+\beta)^{-\alpha}$ corresponding to particular choices $\mu(t)=t-\gamma(\gamma>0), \mu(t)=$ $\delta t(0<\delta<1)$. Such choices are useful in that, when they apply, they give a specific estimate of the rate with which $|u-v|$ approaches 0 . However, the general case depends on the following:

LeMmA 2. If $A>B \geqq 0$ and $\lim _{t \rightarrow \infty} \mu(t)=\infty$, then (17) has a decreasing solution $\sigma$ such that $\lim _{t \rightarrow \infty} \sigma(t)=0$.

The condition on $\mu$ is appropriate, since if $\mu$ is bounded and $B>0$, there can be no such function $\sigma$.

For proof suppose $\lim \mu(t)=\infty$ and construct a sequence $\left\{t_{n}\right\}$ with $t_{0}=0, t_{n+1}>t_{n}+1$, such that $\mu(t)>t_{n-1}+1$ for $t \geqq t_{n}$. For 
$\sigma$ we choose a continuous function which is linear in each interval $\left(t_{n}, t_{n}+1\right)$ and is constant on the complementary intervals. It is readily checked that such a $\sigma$ can be constructed so as to be decreasing and to satisfy (17) except at the corners, and also

$$
0<\sigma\left(t_{n}\right)<(\text { const })\left[\frac{1+B}{1+A}\right]^{n} .
$$

Thus, $\lim \sigma(t)=0$. A modification of this constructions gives a continuously differentiable $\sigma$, but in fact a countable set of hyperplanes $t=$ const. in which $u_{t}-v_{t}$ and $\sigma_{t}$ fail to exist does no harm.

It is perhaps unnecessary to mention that the region and the operator in Theorem 3 can be strongly generalized, by using the full force of Theorem 1 .

11. Boundary conditions of the second kind. The foregoing results extend to cases in which the boundary condition at some points involves only the normal derivative $u_{\nu}$, and not the undifferentiated function $u$. Boundary conditions of this type are of the second kind. A suitable regularity condition is given by the following definition:

DEFinition 5 . The boundary $\Gamma$ is regular if there exists a function $c(x) \in Z^{1}$ satisfying $c_{\nu}(x) \geqq 1$ at all boundary points $(x, t)$ of Type II and also

$$
0 \leqq c \leqq C_{0}, \quad\left|c_{x}\right| \leqq C_{1}, \quad\left|c_{x x}\right| \leqq C_{2} \text { in } G .
$$

The constants $C_{i}$ are called the region constants relative to $c$.

This condition is satisfied by many regions, such as a cube, that do not admit an internally tangent sphere in the sense of Hopf. For further discussions see [7, 9].

We shall consider operators

$$
P_{k} u=u_{t}^{k}-f\left(x, t, u, u_{x}^{k}, u_{x x}^{k} \uparrow, u(\cdot)\right)
$$

subject to the following continuity condition:

$$
\begin{aligned}
& \left(\operatorname{sgn} z_{k}\right)\left[f_{k}\left(x, t, v+z, v_{x}^{k}+p, v_{x x}^{k}+q, v(\cdot)+w(\cdot)\right)\right. \\
& \quad-f\left(x, t, v, v_{x}^{k}, v_{x x}^{k}, v(\cdot)\right) \leqq \omega^{k}\left(t, z,\|w\|_{t},|p|,|q|\right) .
\end{aligned}
$$

Here $\omega^{k}(t, z, s,|p|,|q|)$ is quasimonotone increasing with respect to the arguments $s, z$ and monotone increasing with respect to $|p|,|q|$. For simplicity we assume also that $\omega$ has continuous partial derivatives with respect to each of the arguments $z, s,|p|,|q|$. Instead of introducing a boundary operator $R$ we assume boundary condi- 
tions such that

(18c)

$$
\begin{gathered}
\left|u^{k}-v^{k}\right| \leqq \varepsilon^{k} \\
u^{k}-v^{k}>\varepsilon^{k} \Longrightarrow v_{v}^{k}-u_{v}^{k} \leqq \varepsilon^{k}, \quad v^{k}-u^{k}>\varepsilon^{k} \Longrightarrow u_{v}^{k}-v_{v}^{k} \leqq \varepsilon^{k}
\end{gathered}
$$

at boundary points of type I and II, respectively. Here $\varepsilon$ is a given vector of $R^{m}, \varepsilon>0$.

TheOREM 4. Let (18abc) hold and let $\Gamma$ admit the region constants $C_{0}, C_{1}, C_{2}$. Suppose $\rho$ is an increasing solution of

$$
\rho_{t}^{k} \geqq \omega_{k}\left(t, \rho, \rho, \varepsilon^{k} C_{1}, \varepsilon^{k} C_{2}\right)+\delta^{k}, \quad \rho(0) \geqq\left(1+C_{0}\right) \varepsilon,
$$

on $(0, T]$. Then $\|P u-P v\| \leqq \delta$ in $G$ and $\sup \|u-v\|<\infty$ in $G_{0} \Rightarrow\|u-v\| \leqq \rho$ in $G_{0}$.

The proof follows by use of a comparison function $\psi(x, t)-\tilde{\varepsilon} c(x)$, where $\tilde{\varepsilon}$ is slightly larger than $\varepsilon$ and where $\psi$ is the function used in the proof of Theorem 1. It will be found that the hypothesis involving $p$ and $q$ is needed in a region somewhat larger than that defined by $|p| \leqq C_{1}\left(\max \varepsilon^{k}\right),|q| \leqq C_{2}\left(\max \varepsilon^{k}\right)$.

To illustrate the boundary condition, consider the operator $R$ defined by

$$
R_{k} u=a_{k}(x, t) u^{k}-b_{k}(x, t) u_{\nu}^{k}+g_{k}\left(x, t, u^{k} \uparrow\right)-h_{k}\left(x, t, u_{\nu}^{k} \uparrow\right),
$$

where the arrow denotes monotony as usual. For points $(x, t) \in \Gamma_{0}$ of Type II we require

$$
a_{k}(x, t) \geqq 0, \quad b_{k}(x, t) \geqq 0, \quad a_{k}(x, t)+b_{k}(x, t)=1 .
$$

At points of Type I we set $a_{k}(x, t)=1$ and omit the terms involving $u_{\nu}^{k}$. Then the condition $\|R u-R v\| \leqq \varepsilon$ in $\Gamma_{0}$ implies the boundary hypothesis of Theorem 4.

12. A theorem without growth conditions. For $a \in S^{n}, \widetilde{a} \in$ $S^{n}, \widetilde{b} \in R^{n}, \widetilde{b} \in R^{n}$ it is convenient to define

$$
a \widetilde{a}=\sum_{i, j=1}^{n} a_{i j} \widetilde{a}_{i j}, \quad b \widetilde{b}=\sum_{i=1}^{n} b_{i} \widetilde{b}_{j}, \quad b a b=\sum_{i, j=1}^{n} b_{i} a_{i j} b_{j} .
$$

The hypothesis involving $N|x|^{2}$ and $N|x|$ in Theorem 1 corresponds to classical growth conditions for the coefficients of the linear inequality

$$
u_{t} \leqq a u_{x x}+b u_{x}, \quad a \geqq 0, \quad m=1
$$

where $a=a(x, t) \in S^{n}, b=b(x, t) \in \boldsymbol{R}^{n}$ and the products $a u_{x x}, b u_{x}$ denote contractions as above. Namely, the maximum principle holds for 
bounded solutions in unbounded regions if $a=O\left(|x|^{2}\right)$ and $b=O(|x|)$, uniformly in $t$, as $|x| \rightarrow \infty$. It was noticed in [10] that these growth conditions can be dropped if the elliptic problem associated with $a u_{x x}+b u_{x}=0$ has effective dimension $\leqq 2$ in the sense of Meyers and Serrin [3]; see Definition 6 below. Conversely, the latter hypothesis is essential for the validity of the maximum principle in the problem

$$
u_{t} \leqq \lambda\left(a u_{x x}+b u_{x}\right), \quad \sup u<\infty, \quad m=1
$$

when $G=\Omega \times(0, T], \boldsymbol{R}^{n}-\Omega$ is bounded, and the growth of the function $\lambda: G \rightarrow \boldsymbol{R}_{+}$is unrestricted.

By means of the technique used in the proof of Theorem 1 these results can be generalized to nonlinear systems containing functionals. To this end, we introduce the following definition:

Definition 6. Let $a$ and $b$ denote functions $G \rightarrow S^{n}, G \rightarrow R^{n}$, respectively. The operator $S$ defined for $u \in Z^{1}$ by $S u=a u_{x x}+b u_{x}$ is said to be of Meyers-Serrin type if $a \geqq 0$ and for some $r_{0}$

$$
|x|^{2} \frac{\operatorname{trace} a(x, t)+b(x, t) x}{x a(x, t) x} \leqq 2+\phi(|x|), \quad(x, t) \in G, \quad|x|>r_{0}
$$

where $\phi$ is a continuous function $\boldsymbol{R} \rightarrow \boldsymbol{R}$ satisfying

$$
\int_{r_{0}}^{\infty} \frac{1}{r} \exp \left[-\int_{r_{0}}^{r} \frac{\phi(s)}{s} d s\right] d r=\infty
$$

The importance of this condition is that, when it holds, the equation $S y \leqq 0$ has a radial solution $y=\mu(|x|)>0$ for $|x|>r_{0}$ which satisfies $\lim u(\tau)=\infty$ as $\tau \rightarrow \infty$ : see [3].

We consider operators of the form

$$
P_{k} u=u_{t}^{k}-f_{k}\left(x, t, u, S_{k} u^{k} \uparrow, u(\cdot)\right)
$$

where $f_{k}$ is a function $G \times \boldsymbol{R}^{m} \times \boldsymbol{R} \times Z^{m} \rightarrow \boldsymbol{R}$ and where each $S_{k}$ is an operator of Meyers-Serrin type. As continuity condition we require

$$
\begin{gathered}
\left(\operatorname{sgn} z^{k}\right)\left[f_{k}\left(x, t, v+z, S_{k} v^{k}, v(\cdot)+w(\cdot)\right)-f_{k}\left(x, t, v, S_{k} v^{k}, v(\cdot)\right)\right] \\
\leqq \omega_{k}\left(t,\|z\|,\|w\|_{t}\right),
\end{gathered}
$$

where $\omega$ satisfies the same conditions as in Theorem 1. Namely, $\omega$ is quasimonotone increasing and

$$
\omega(t, y+z, y+\bar{z})-\omega(t, y, y) \leqq K(|z|+|\bar{z}|) \quad(z \geqq 0, \bar{z} \geqq 0) .
$$

The difference between this hypothesis and that of Theorem 1 is 
that here we have no restriction involving the arguments $p$ and $q$, which correspond to the dependence of $f$ on $u_{x}$ and $u_{x x}$, respectively.

THEOREM 5. Let (20abc) hold in a bounded or unbounded region $G$ and suppose $\rho$ is an increasing solution of

$$
\rho_{t} \geqq \omega(t, \rho, \rho)+\dot{\delta}, \quad \rho \geqq \varepsilon, \quad 0<t \leqq T .
$$

Then

$$
\begin{gathered}
\|P u-R v\| \leqq \delta \text { in } G,\|u-v\| \leqq \varepsilon \text { in } \Gamma, \sup _{G_{0}}\|u-v\|<\infty \\
\Longrightarrow\|u-v\| \leqq \rho \text { in } G_{0} .
\end{gathered}
$$

If $G=\Omega \times(0, T]$, where $\boldsymbol{R}^{n}-\Omega$ is bounded, the hypothesis that each $S_{k}$ is of Meyers-Serrin type is essential even when $f$ is linear and the arguments $u, u(\cdot)$ do not occur. This follows from the above discussion of (19); cf. [10].

To prove Theorem 5 construct radial functions $y_{k}(x)=Y_{k}(|x|)$ such that $S_{k} y_{k} \leqq 0$ for large $|x|$ and also $\lim y_{k}(x)=\infty$ for $|x| \rightarrow \infty$. We choose $r_{0}$ so large that every $y_{k}$ is positive (and satisfies its differential inequality) for $|x|>r_{0}$, and we define $y_{k}(x)=0$ for $|x| \leqq r_{0}$. Theorem 5 now follows by use of a comparison function

$$
\psi(x, t)=\alpha y(x)+\lambda_{i}(t) \sigma(t)+\rho(t)
$$

with $y=\left(y_{1}, y_{2}, \cdots, y_{m}\right)$ and with $\lambda_{i}$ and $\sigma$ as in the proof of Theorem 1 . In the present case the boundary is ruled out automatically as a possible location of the Nagumo point, and in other respects the proof parallels the proof of Theorem 1 .

\section{REFERENCES}

1. L. Collatz, Aufgaben monotoner Art, Arch. Math., 3 (1952), 366-376.

2. V. Lakshmikantham and S. Leela, Differential and Integral Inequalities, Vol. I and II. Academic Press, New York and London, 1969.

3. N. Meyers and J. Serrin, The exterior Dirichlet problem for second order elliptic partial differential equations, J. Math. Mech., 9 (1960), 513-538.

4. K. Nickel, Fehlerschranken und Eindeutigkeitsaussagen für die Lösungen nichtlinearer, stark gekoppelter, parabolischer Differentialgeichungen, to appear in Math. Z. 5. - New results on strongly coupled systems of parabolic differential equations, Proceedings of the Fourth Dundee Conference on Ordinary and Partial Differen. tial Equations, March 30-April 2, 1976. Lecture Notes in Mathematics, Vol. 564 Springer-Verlag, Berlin-Heidelberg-New York, 1976. This paper is a revision of [4] above.

6. R. Redheffer, Differentialungleichungen unter schwachen Voraussetzungen, Abhandl. Math. Sem. Univ. Hamburg, 41 (1967), 33-50.

7. _ Die Collatzsche Monotonie bei Anfangswertproblemen, Arch. Rat. Mech. and Anal., 14 (1963), 196-212.

8. R. Redheffer and W. Walter, Existence theorems for strongly coupled systems of 
partial differential equations over Bernstein classes, Bull. Amer. Math. Soc., 82, 6 (1976), 899-902.

9. R. Redheffer and W. Walter, Uniqueness, Stability and Error Estimation for Parabolic Functional-differential Equations, Ber. 9, Univ. Karlsruhe (1976). To be published in the Jubilee Volume dedicated to the 70th anniversary of Academician I. N. Vekua in 1977.

10. —, Das Maximumprinzip in unbeschränkten Gebieten für parabolische Ungleichungen mit Funktionalen, Math. Ann., 226 (1977), 155-170.

11. — Inequalities involving derivatives, to appear in Pacific J. Math.

12. J. Szarski, Differential inequalities, Monografie Matematyczne, Tom 43. Warszawa, 1965.

13. W. Walter, Differential and integral inequalities, Ergebnisse der Mathematik und ihrer Grenzgebiete, Bd, 55, Springer-Verlag, 1970.

Received July 17, 1978. The first author was supported by NSF Grant No. MCS7703570 .

University OF CALIFornia

Los ANGeles, CA90024

AND

UNIVERSITY OF KARLSRUHE

KarlSRUhe, Germany 


\section{PACIFIC JOURNAL OF MATHEMATICS}

\section{EDITORS}

DONALD BABBITT (Managing Editor)

University of California

Los Angeles, CA 90024

HUgo RossI

University of Utah

Salt Lake City, UT 84112

C. C. Moore and ANDrew OGG

University of California

Berkeley, CA 94720

\section{J. DUGUNDJI}

Department of Mathematics

University of Southern California

Los Angeles, CA 90007

R. FinN and J. MILGRaM

Stanford University

Stanford, CA 94305

\section{ASSOCIATE EDITORS}
E. F. BECKENBACH
B. H. NeumanN
F. WOLF
K. YOSHIDA

\section{SUPPORTING INSTITUTIONS}

UNIVERSITY OF BRITISH COLUMBIA CALIFORNIA INSTITUTE OF TECHNOLOGY

UNIVERSITY OF CALIFORNIA

MONTANA STATE UNIVERSITY

UNIVERSITY OF NEVADA, RENO

NEW MEXICO STATE UNIVERSITY

OREGON STATE UNIVERSITY

UNIVERSITY OF OREGON
UNIVERSITY OF SOUTHERN CALIFORNIA

STANFORD UNIVERSITY

UNIVERSITY OF HAWAII

UNIVERSITY OF TOKYO

UNIVERSITY OF UTAH

WASHINGTON STATE UNIVERSITY

UNIVERSITY OF WASHINGTON

The Supporting Institutions listed above contribute to the cost of publication of this Journal, but they are not owners or publishers and have no responsibility for its content or policies.

Mathematical papers intended for publication in the Pacific Journal of Mathematics should be in typed form or offset-reproduced, (not dittoed), double spaced with large margins. Please do not use built up fractions in the text of the manuscript. However, you may use them in the displayed equations. Underline Greek letters in red, German in green, and script in blue. The first paragraph or two must be capable of being used separately as a synopsis of the entire paper. Please propose a heading for the odd numbered pages of less than 35 characters. Manuscripts, in triplicate, may be sent to any one of the editors. Please classify according to the scheme of Math. Reviews, Index to Vol. 39. Supply name and address of author to whom proofs should be sent. All other communications should be addressed to the managing editor, or Elaine Barth, University of California, Los Angeles, California, 90024.

50 reprints to each author are provided free for each article, only if page charges have been substantially paid. Additional copies may be obtained at cost in multiples of 50 .

The Pacific Journal of Mathematics is issued monthly as of January 1966. Regular subscription rate: $\$ 84.00$ a year (6 Vols., 12 issues). Special rate: $\$ 42.00$ a year to individual members of supporting institutions.

Subscriptions, orders for numbers issued in the last three calendar years, and changes of address should be sent to Pacific Journal of Mathematics, P.O. Box 969, Carmel Valley, CA 93924, U.S.A. Older back numbers obtainable from Kraus Periodicals Co., Route 100, Millwood, NY 10546.

PUBLISHED BY PACIFIC JOURNAL OF MATHEMATICS, A NON-PROFIT CORPORATION

Printed at Kokusai Bunken Insatsusha (International Academic Printing Co., Ltd.). 8-8, 3-chome, Takadanobaba, Shinjuku-ku, Tokyo 160, Japan.

Copyright (C) 1979 by Pacific Journal of Mathematics Manufactured and first issued in Japan 


\section{Pacific Journal of Mathematics}

\section{Vol. 85, No. $2 \quad$ October, 1979}

Charles A. Akemann and Steve Wright, Compact and weakly compact derivations of $C^{*}$-algebras ........................... 253

Dwight Richard Bean, Andrzej Ehrenfeucht and George Frank McNulty, Avoidable patterns in strings of symbols ................... 261

Richard Clark Brown, Notes on generalized boundary value problems in Banach spaces. I. Adjoint and extension theory.................

Kenneth Alexander Brown and John William Lawrence, Injective hulls of group rings .................................... 323

Jacob Burbea, The Schwarzian derivative and the Poincaré metric ....... 345

Stefan Andrus Burr, On the completeness of sequences of perturbed polynomial values ....................................

Peter H. Chang, On the characterizations of the breakdown points of quasilinear wave equations..............................

Joseph Nicholas Fadyn, The projectivity of $\operatorname{Ext}(T, A)$ as a module over

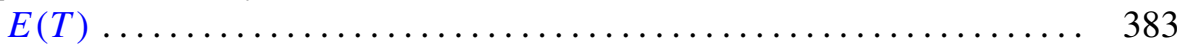

Donald Eugene Maurer, Arithmetic properties of the idèle discriminant .... 393

Stuart Rankin, Clive Reis and Gabriel Thierrin, Right subdirectly irreducible semigroups ................................. 403

David Lee Rector, Homotopy theory of rigid profinite spaces. I ........ 413 Raymond Moos Redheffer and Wolfgang V. Walter, Comparison theorems for parabolic functional inequalities................

H. M. (Hari Mohan) Srivastava, Some generalizations of Carlitz's theorem

James Alan Wood, Unbounded multipliers on commutative Banach algebras....

T. Yoshimoto, Vector-valued ergodic theorems for operators satisfying norm

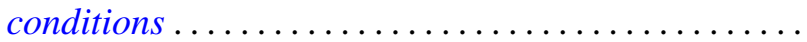

Jerry Searcy and B. Andreas Troesch, Correction to: "A cyclic inequality and a related eigenvalue problem".

Leslie Wilson, Corrections to: "Nonopenness of the set of Thom-Boardman maps" 